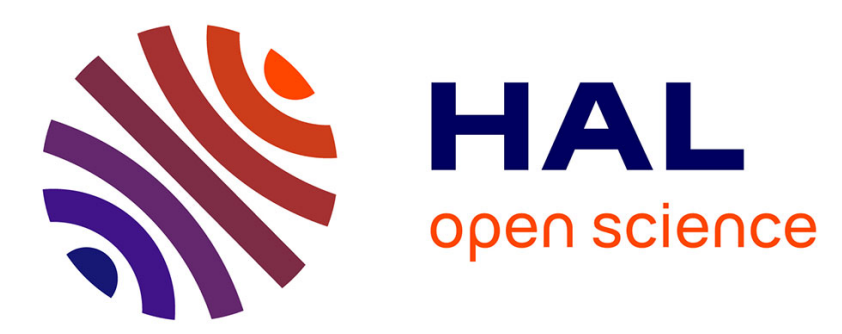

\title{
Magnetic Flattening of Stem-Cell Spheroids Indicates a Size-Dependent Elastocapillary Transition
}

\author{
Francois Mazuel, Myriam Reffay, Vicard Du, Jean-Claude Bacri, Jean-Paul
} Rieu, Claire Wilhelm

\section{- To cite this version:}

Francois Mazuel, Myriam Reffay, Vicard Du, Jean-Claude Bacri, Jean-Paul Rieu, et al.. Magnetic Flattening of Stem-Cell Spheroids Indicates a Size-Dependent Elastocapillary Transition. Physical Review Letters, 2015, 114, pp.098105. 10.1103/PhysRevLett.114.098105 . hal-02307262

\section{HAL Id: hal-02307262 https://univ-lyon1.hal.science/hal-02307262}

Submitted on 16 Feb 2021

HAL is a multi-disciplinary open access archive for the deposit and dissemination of scientific research documents, whether they are published or not. The documents may come from teaching and research institutions in France or abroad, or from public or private research centers.
L'archive ouverte pluridisciplinaire HAL, est destinée au dépôt et à la diffusion de documents scientifiques de niveau recherche, publiés ou non, émanant des établissements d'enseignement et de recherche français ou étrangers, des laboratoires publics ou privés. 


\title{
Magnetic Flattening of Stem-Cell Spheroids Indicates a Size-Dependent Elastocapillary Transition
}

\author{
Francois Mazuel, ${ }^{1}$ Myriam Reffay, ${ }^{1}$ Vicard Du, ${ }^{1}$ Jean-Claude Bacri, ${ }^{1}$ Jean-Paul Rieu, ${ }^{2}$ and Claire Wilhelm ${ }^{1, *}$ \\ ${ }^{1}$ Laboratoire Matière et Systèmes Complexes, UMR 7057, CNRS, Université Paris Diderot, 75013 Paris, France \\ ${ }^{2}$ Institut Lumière Matière, UMR 5306, Université Lyon 1-CNRS, Université de Lyon, 69622 Villeurbanne cedex, France
}

(Received 24 July 2014; revised manuscript received 5 December 2014; published 4 March 2015)

\begin{abstract}
Cellular aggregates (spheroids) are widely used in biophysics and tissue engineering as model systems for biological tissues. In this Letter we propose novel methods for molding stem-cell spheroids, deforming them, and measuring their interfacial and elastic properties with a single method based on cell tagging with magnetic nanoparticles and application of a magnetic field gradient. Magnetic molding yields spheroids of unprecedented sizes (up to a few $\mathrm{mm}$ in diameter) and preserves tissue integrity. On subjecting these spheroids to magnetic flattening (over 150g), we observed a sizedependent elastocapillary transition with two modes of deformation: liquid-drop-like behavior for small spheroids, and elastic-sphere-like behavior for larger spheroids, followed by relaxation to a liquidlike drop.
\end{abstract}

DOI: 10.1103/PhysRevLett.114.098105

The mechanical properties of biological tissues have become a major focus of research with immediate implications for morphogenesis [1,2], and cancer [3]. One of the earliest tissue models is the multicellular spheroid. Their three-dimensional cellular organization gives to these spheroids specific properties and functions that mimic many in vivo characteristics, such as spatial cell-to-cell interactions, and oxygen, nutrient, and metabolite gradients [4]. They have been used to study embryonic tissues [5], as a tumor model system [6], and for drug screening [7].

Experiments and simulations with spheroids, combined with concepts from soft-matter physics, have helped to unravel the properties of these biological objects which variously round up, fuse, or sort out [8,9]. Steinberg was the first to draw an analogy between embryonic tissues and fluids $[10,11]$. He went on to formulate the differential adhesion hypothesis, whereby cell-cell adhesion is responsible for tissue surface tension [12]. More recent variants of this hypothesis take into account the contribution of the actin-myosin contractile network to the surface tension of cellular spheroids [13-15]. Spheroids have also been reported to behave as elastic solids at short time scales (seconds to minutes) and as very viscous fluids at long time scales (minutes to hours) [16,17], with cellular reorganizations (cell-cell rearrangements) during this transition [18]. Interestingly, the elastic properties of spheroids are mainly due to cellular elasticity, and spheroids hence represent a tool for probing the properties of thousands of cells at the same time [19]. Several models (agent-based and continuous) have been proposed to describe the mechanics of spheroids and growing tumors [18,20-22], but more experimental data are needed to validate them.

Few experimental methods are suitable for measuring the interfacial and rheological properties of spheroids.
PACS numbers: 87.19.R-, 87.18.Ed, 87.18.Fx, 75.50.Mm

The compression-plate tensiometer [23,24] in which an aggregate is deformed between two parallel plates, derives the spheroid's surface tension from its shape and the compressive force at equilibrium. Micropipette aspiration of a spheroid can also be used to determine its surface tension, elastic constant, and viscosity, but only at a local level [25]. Finally, the centrifugation force tensiometer $(100 \mathrm{~g})$ links spheroid flattening to its surface tension based on the equilibrium shape [26]. However, the spheroid first needs to be fixed, which may influence its shape and rules out dynamic observation of its compression (inside the centrifuge) and relaxation. Here we used a magnetic force tensiometer as a simple alternative to the parallel plate and centrifugation tensiometers to deform magnetically labeled spheroids and study their mechanical properties. This magnetic approach can be used to measure the surface tension, elastic modulus, and viscosity of cellular spheroids.

The radius of spheroids so far produced has never exceeded $150 \mu \mathrm{m}$, a limit imposed by the different production techniques [27] (pending drops, micropatterned microwells or surfaces, low-speed centrifugation or shaking culture) and by the use of nutrient-dependent cells (nutrient diffusion is poor at the core of the spheroid). Here we also used magnetic force to assemble cells into spheroids of controlled size. We developed a magnetic molding technique yielding spheroids of unprecedented sizes. Combined with magnetic flattening of the spheroids thus obtained, we were able to explore the effect of spheroid size on the mechanical response and observed a size-dependent transition: below a critical radius the spheroids did not experience an elastic regime, whereas above this radius they went through an elastic-to-liquid transition.

We chose to use human mesenchymal pluripotent stem cells (MSC, Lonza), which can be induced to interact with 
one another by placing them in an aggregation medium such as that used to induce differentiation into cartilage [28]. These cells readily handle nutrient starvation $[29,30]$ and are therefore capable of forming large and viable spheroids. They were magnetized by adding iron oxide nanoparticles ( $7 \mathrm{~nm}$ maghemite particles) to the culture medium (1 hour at $[\mathrm{Fe}]=10^{-3} \mathrm{moll}^{-1}$ ). These nanoparticles are taken up by the endocytic pathway (26 pg of iron per cell), and do not affect the cells' behavior [31]. In particular, the cells' differentiation capacity is unaffected, either by nanoparticle incorporation [32], or by the magnetic field [28]. Moreover, the nanoparticles are confined within endosomes (diameter $d_{\text {endo }} \simeq 1 \mu \mathrm{m}$, and magnetic moment $m_{\text {endo }} \simeq 10^{-15} \mathrm{~A} \mathrm{~m}^{2}$, Supplemental Material, Fig. S1 [33]). These endosomes are mobilized by molecular motors, and the resulting effective temperature (about 1000-fold the bath temperature [34]) prevents any potential mechanosensitive endosomal pulling $\left(k_{B} T_{\text {eff }} \simeq 10^{-17} \mathrm{~J} \gg F_{\text {endo }} d_{\text {endo }} \simeq 2 \times 10^{-19} \mathrm{~J}\right)$.

The magnetic cells were magnetically compacted, within spherical molds (between 1 and $3 \mathrm{~mm}$ in diameter) prepared in agarose gel. The mold manufacturing process is described in Fig. 1(a). The larger the mold, the larger the number of deposited cells [33]. The final diameters of the spheroids were smaller than their initial diameters, showing that the cells still contracted in these conditions [35]. This technique yields spherical spheroids with a wide range of diameters [Fig. 1(b)].

Before using these magnetic spheroids, it was necessary to establish that the cells remained viable at the center of the structure. Confocal microscopy of cryotome sections prepared from the largest spheroids 20 hours after molding [Fig. 1(c) and Fig. S2 for confocal microscopy pictures] revealed only viable cells throughout the spheroid, by contrast with a negative control produced in the same molding conditions but in which cell death was induced by holding the temperature at $45^{\circ} \mathrm{C}$ for $30 \mathrm{~min}$ (Fig. S3). This good survival can be explained by the cells' resistance to nutrient starvation and their short maturation time. The nanoparticles were homogeneously distributed within the spheroids [Fig. 1(d)], providing uniform magnetization. Magnetophoresis (migration of spheroids across a uniform magnetic field gradient in a viscous liquid, $\mathrm{Re} \simeq 10^{-4}$ ) showed that the bulk magnetization of the spheroids was independent of their size, with an average value of $M_{V}=$ $472 \pm 110 \mathrm{~A} \mathrm{~m}^{-1}$.

To prepare the magnetic force tensiometer, a cylindrical neodymium magnet $(6 \mathrm{~mm}$ high and $6 \mathrm{~mm}$ in diameter) was selected to generate a homogenous magnetic force per unit volume $\left(f_{V}=M_{V} \times \operatorname{grad} B\right)$. Close to the surface (magnetic field of $470 \mathrm{mT}$ ) and within a cylindric volume $2 \mathrm{~mm}$ high and $2 \mathrm{~mm}$ in diameter, the magnetic field gradient was uniform $\left(\operatorname{grad} B=230 \mathrm{~T} \mathrm{~m}^{-1}\right)$. Spheroid flattening was monitored in a home-made $37^{\circ} \mathrm{C}$ thermostated container enclosed by glass slides at the bottom (in direct contact

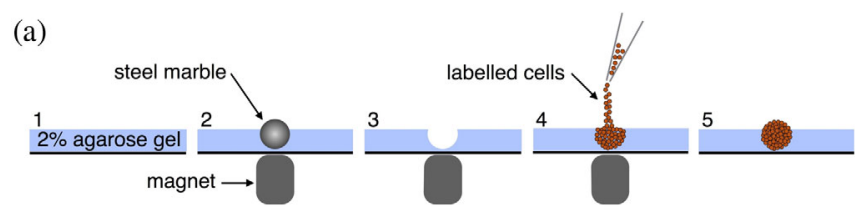

(b)

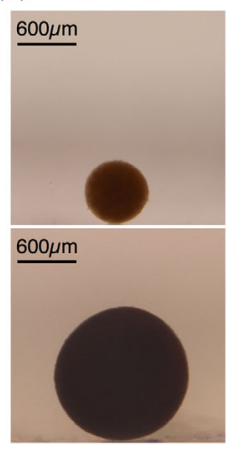

(c)

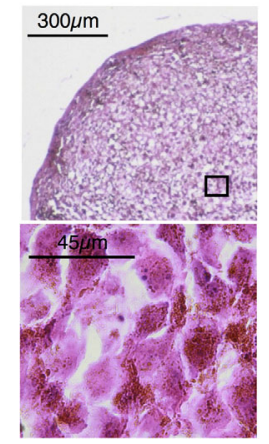

(d)

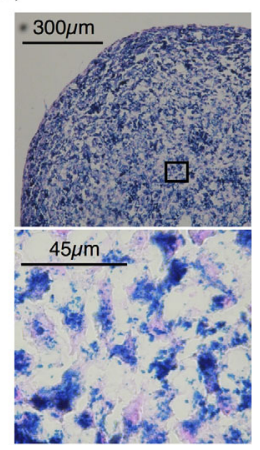

FIG. 1 (color online). (a) Magnetic molding method to form spheroids: (1) a thin layer of $2 \%$ agarose solution is poured into a culture dish. (2) A cylindrical magnet (1 mm diameter) is placed under the dish as the agarose is still liquid to attract a steel marble of calibrated diameter. (3) The agarose solidifies as it cools down, the marble is removed forming an hemispherical mold. The surface of the mold is treated with an anti-adhesive solution of PLL-PEG. (4) The culture dish is filled with aggregation medium. The magnetically labeled cells are deposited in a small volume above the well. The magnet attracts the cells and compacts them. It is removed after 30 seconds. (5) The dish is placed in an incubator $\left(37^{\circ} \mathrm{C}\right)$. Within 20 hours the aggregate is spherical. (b) Typical spheroid images $20 \mathrm{~h}$ after molding. (c) Section of a spheroid stained with hematoxylin and eosin that colors cytoplasm in pink and nucleus in purple around 20 hours after molding. The morphology is characteristic of cells in good condition. (d) Section of a spheroid stained with Perls reagent that colors iron in blue.

with the magnet) and on the sides (allowing camera imaging) (Fig. S4).

To validate the approach, ferrofluid drops (aqueous suspension of the nanoparticles used for cell labeling) immersed in silicon oil and deposited on a silanized glass slide (nonwetting conditions) were flattened [Fig. 2(a)], at various drop volumes (1-5 $\mu \mathrm{l})$ and iron concentrations (125-500 mM) (Fig. S5). When placed right above the magnet, the ferrofluid drops deformed instantaneously from their initial spherical shape to an equilibrium shape (temporal evolution in Fig. S6A). The equilibrium profile is driven by the balance between surface tension $(\gamma)$ and force per unit volume $\left(f_{V}\right)$. The exact profile of the drops in nonwetting conditions can be obtained by numerically integrating the Laplace law for capillarity. The shapes of the ferrofluid drops at equilibrium perfectly fitted the Laplace profile [Fig. 2(a)]. The average surface tension was $\gamma=54 \pm 1 \mathrm{mN} \mathrm{m}^{-1}$, in good agreement with the classical sessile drop experiment performed with bigger drops (Fig. S7). 
(a)

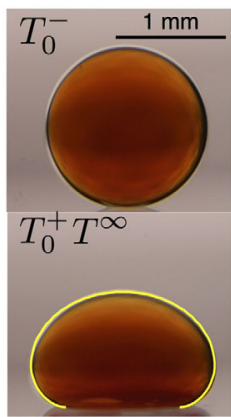

(b)

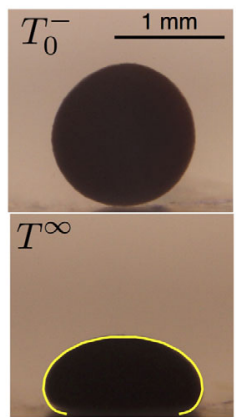

(c)

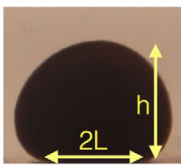

(d)

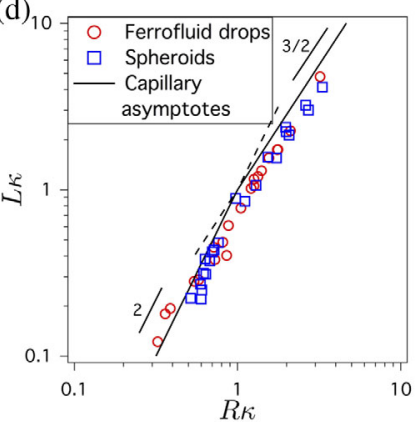

(e)

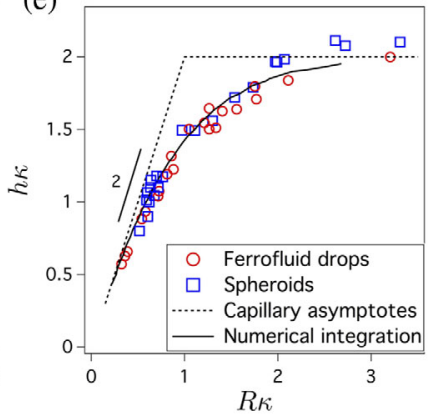

FIG. 2 (color online). (a) Ferrofluid drop before flattening $\left(T_{0}^{-}\right)$ and under magnetic flattening $\left(T_{0}^{+}=10 \mathrm{~s}\right.$, same image at $T^{\infty}=1 \mathrm{~h}$ ). The Laplace capillary profile integrated numerically with Matlab's function ODE45 is superimposed (yellow line) on the image. (b) Spheroid before flattening $\left(T_{0}^{-}\right)$and at equilibrium under magnetic flattening force $\left(T^{\infty}\right)$, with Laplace capillary profile in yellow. (c) Definition of the deformation parameters: the height $(h)$ and the radius of the contact zone $(L)$. (d), (e) Radius of the contact zone (d) and height (e) of ferrofluid drops and spheroids at equilibrium $\left(T^{\infty}\right)$ as a function of their initial radius. All lengths are normalized by the capillary length $\kappa^{-1}$. Asymptotic capillary laws and numerical integration of capillary laws are shown in both plots.

Magnetic cellular spheroids were then deformed in the same conditions [Fig. 2(b)], corresponding to a mean magnetic force per unit volume of $f_{V}=(1.2 \pm 0.14) \times$ $10^{5} \mathrm{~N} \mathrm{~m}^{-3}(150 \mathrm{~g})$. The glass substrate was coated with PLL-PEG to prevent cells from adhering to it (nonwetting conditions). All the spheroids reached an equilibrium shape after $T^{\infty}=1$ hour of magnet application (Fig. S6), correctly fitting the Laplace profile [Fig. 2(b)] and providing a surface tension of $\gamma=18 \pm 7 \mathrm{mN} \mathrm{m}^{-1}$ (average for 13 spheroids) independent of the spheroid size. Magnetic flattening of droplets or spheroids, therefore, appears to be a reliable tool for measuring their surface tension.

Each flattened spheroid can further be described by a set of three lengths [Fig. 2(c)]: $L$ (radius of the contact zone), $h$ (spheroid height) and $R$ (initial spheroid radius). These lengths have to be compared with the capillary length $\kappa^{-1}=\left(\gamma / f_{v}\right)^{1 / 2}=390 \mu \mathrm{m}$. Figures 2(d) and 2(e) show $L \kappa$ and $h \kappa$ as a function of $R \kappa$. Capillary laws govern the asymptotic behaviors of $L_{\text {cap }} \kappa$ and $h_{\text {cap }} \kappa$ [36]. For $R \ll \kappa^{-1}$, surface tension predominates and the spheroids remain spherical. The height $h_{\text {cap }}^{s}$ of the spheroid is $2 R\left(h_{\text {cap }}^{s} \kappa=\right.$ (a)

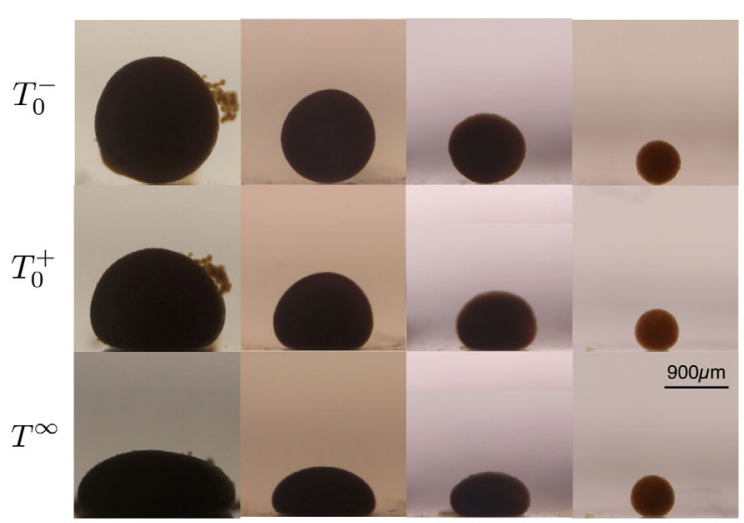

(b)

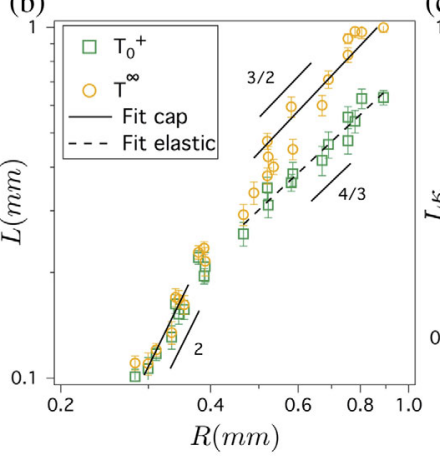

(c)

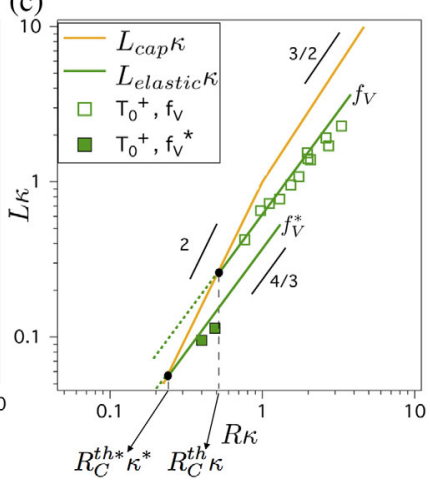

FIG. 3 (color online). (a) Cellular spheroids before flattening $\left(T_{0}^{-}\right)$, under magnetic flattening $\left(T_{0}^{+}=10 \mathrm{~s}\right.$ after the force application), and after 1 hour $\left(T^{\infty}\right)$. (b) Radius of the contact zone $(L)$ as a function of the aggregate's initial radius $(R) 10$ seconds after the application of the magnetic force $\left(T_{0}^{+}\right)$and at equilibrium $\left(T^{\infty}\right)$. (c) Role of $f_{V}$ in the elastocapillary transition. For the capillary regime the two orange straight lines correspond to the two asymptotic behaviors $\left[L_{\text {cap }}^{s} \kappa \propto(R \kappa)^{2}\right.$ and $L_{\text {cap }}^{p} \kappa \propto$ $\left.(R \kappa)^{3 / 2}\right] . L_{\text {cap }} \kappa$ is independent of $f_{V}$. The predicted elastic deformation $L_{\text {elastic }} \kappa=\left[\left(1-\sigma^{2}\right) \pi / E\right]^{1 / 3} f_{V}^{1 / 6} \gamma^{1 / 6}(R \kappa)^{4 / 3}$ is plotted for the two $f_{V}$ considered $\left(f_{V}^{*}<f_{V}\right)$. In the $R<R_{C}$ region, the dotted line represents the deformation that the spheroid would have reached if it was purely elastic, here inaccessible because the final deformation is imposed by the surface tension. For means of clarity, only experimental data at $R>R_{C}$ and $T_{0}^{+}$(full and open squares) are presented (they are slightly different to those shown in Fig. 3(b), because each is normalized by its corresponding capillary length and not the mean capillary length).

$2 R \kappa)$, while the size of the contact zone $L_{\text {cap }}^{s}$ increases as $L_{\text {cap }}^{s} \simeq \sqrt{2 / 3} R^{2}\left(f_{V} / \gamma\right)^{1 / 2}$, that is $L_{\text {cap }}^{s} \kappa \propto(R \kappa)^{2}$. Above the capillary length, $R \gg \kappa^{-1}$, surface tension is negligible, leading to a puddle shape with a constant height $\left(h_{\text {cap }}^{p}=\right.$ $2 \kappa^{-1}$ ) and a contact length $L_{\text {cap }}^{p}$ varying as $L_{\text {cap }}^{p} \simeq$ $\sqrt{2 / 3} R^{3 / 2}\left(f_{V} / \gamma\right)^{1 / 4}$, that is $L_{\text {cap }}^{p} \propto \propto(R \kappa)^{3 / 2}$. The experimental data perfectly matched these predicted behaviors (at $\left.T^{\infty}\right): L_{\text {cap }} \kappa$ depends on $(R \kappa)^{2}$ below the capillary length and on $(R \kappa)^{3 / 2}$ above it. In addition, $h_{\text {cap }} \kappa$ was in good agreement with the exact numerical integration of the Laplace equation. This confirms the liquidlike hypothesis at long time scales. 
Is there then any regime in which spheroid elasticity predominates? Whereas the change from the initial to equilibrium shape was instantaneous for ferrofluid drops, spheroids displayed time-dependent behaviors that varied with their size [Fig. 3(a)]. At short time scales $\left(T_{0}^{+} \simeq 10 \mathrm{~s}\right.$ ), both small and large spheroids underwent reversible deformation. At longer time scales, the profile of the small spheroids no longer evolved, while the larger spheroids continued to deform irreversibly until they reached their equilibrium shape $\left(T^{\infty}=1 \mathrm{~h}\right)$.

The effect of the spheroid's size on the mechanical response is illustrated in Fig. 3(b), where $L$ at $T_{0}^{+}$and $T^{\infty}$ is plotted as a function of the initial radius $R$. A critical radius $R_{C}$ (between 350 and $400 \mu \mathrm{m}$ ) clearly forms a frontier between two behaviors. For $R<R_{C}$, the deformation of the spheroid at $T_{0}^{+}$and $T^{\infty}$ is the same. For $R>R_{C}, L$ initially (at $T_{0}^{+}$) varies as $R_{0}^{4 / 3}$, corresponding to purely elastic solid behavior, as described by the Hertz theory for an elastic sphere of initial radius $R$, elastic modulus $E$, and Poisson ratio $\sigma(=1 / 2): L_{\text {elastic }}=\left[\left(1-\sigma^{2}\right) \pi / E\right]^{1 / 3} f_{V}^{1 / 3} R^{4 / 3}$. The fitting of the elastic behavior provides the elastic modulus of the spheroids: $E=660 \pm 120 \mathrm{~Pa}$. This value is similar to values reported for murine sarcoma cell lines [25] and embryonic liver tissue [17], as well as the individual Young's modulus of MSC [37]. At longer times $T^{\infty}, L$ varies as $R^{3 / 2}$, reflecting liquidlike behavior.

Spheroids have two ways of deforming [17] before reaching their capillarity-driven equilibrium shape: first, rapid elastic deformation of individual cells in the aggregate, then a more viscouslike response where cells rearrange. These two regimes of deformation are clearly reflected by the temporal evolution of the spheroid height, which follows a two-exponential times law (Fig. S6) with a first characteristic time around $10 \mathrm{~s}$ and the largest one of $1200 \pm 150 \mathrm{~s}$. The effective viscosity extracted from the long-time viscous behavior thus writes $\eta=E \tau=(7.9 \pm 2.4) \times 10^{5} \mathrm{~Pa}$ s. This estimate is in agreement with other values of cellular spheroid viscosities $[14,25]$.

Let us now focus on the critical radius $R_{C}$, which delineates the capillary-to-elastic transition at short times $\left(T_{0}^{+}\right)$. The maximal spheroid's deformation length is imposed by $L_{\text {cap. }}$. For spheroids with an initial radius smaller than $R_{C}$, the rapid elastic deformation is sufficient to immediately reach the equilibrium shape $\left(L_{\text {elastic }}>L_{\text {cap }}\right)$, whereas cell rearrangements are required for spheroids with an initial radius larger than $R_{C}$ ( $\left.L_{\text {elastic }}<L_{\text {cap }}\right) . \quad R_{C}$ is thus defined by equalling $L_{\text {elastic }}=L_{\text {cap }}$. As the transition occurs slightly below $\kappa^{-1}$ (spherical case) in the present system, we consider only $L_{\text {cap }}^{s}$ (the puddle case $L_{\text {cap }}^{p}$ is commented on Fig. S8), and we can derive $R_{C}^{\text {th }} \simeq(3 / 2)^{3 / 4}\left[\left(1-\sigma^{2}\right) \pi /\right.$ $E]^{1 / 2} \gamma^{3 / 4} f_{V}^{-1 / 4}$. In addition to the experimental data obtained at $f_{V}=1.2 \times 10^{5} \mathrm{~N} \mathrm{~m}^{-3}$, we performed a second series of experiments at a smaller force $\left(f_{V}^{*}=f_{V} / 12=\right.$ $\left.(1 \pm 0.1) \times 10^{4} \mathrm{~N} \mathrm{~m}^{-3}, \quad \kappa^{-1 *}=1350 \mu \mathrm{m}\right) \quad$ obtained by reducing the cellular iron dose (cell incubation at $[\mathrm{Fe}]=1.5 \times 10^{-4} \mathrm{moll}^{-1}$ for $30 \mathrm{~min}$ ). For such lower force, we could not cover a wide range of sizes as the deformation was only measurable for large spheroids. Figure 3(c) presents the theoretically predicted deformation regimes: capillary $L_{\text {cap }}^{S} \kappa(R \kappa)$ and elastic $L_{\text {elastic }} \kappa(R \kappa)$ for these two forces $\left(f_{V}, f_{V}^{*}\right)$. The experimental $L$ values obtained at $\left(f_{V}, T_{0}^{+}\right)$and $\left(f_{V}^{*}, T_{0}^{+}\right)$, also plotted in Fig. 3(c), are in good agreement with the theoretical predictions. To conclude, a 12-fold decrease in $f_{V}$ results in a twofold increase in $R_{C}$, from $R_{C}^{\mathrm{th}}=213 \pm 90 \mu \mathrm{m}$ at $f_{V}$ to $R_{C}^{\text {th } *}=398 \pm 166 \mu \mathrm{m}$ at $f_{V}^{*}$. The predicted $R_{C}^{\text {th }}$ at $f_{V}$ is within the same range as the experimental value shown in Fig. 3(b) (the small difference is probably due to the fact that the transition happens close to the $\kappa^{-1}$ zone). For information, gravity alone would lead to a critical radius $R_{C}^{\text {th }}(1 g) \sim 800 \mu \mathrm{m}$.

In conclusion, we placed magnetic nanoparticles inside stem cells, giving them magnetic properties without affecting their functions. We developed a magnetic molding technique capable of creating spheroids with a wide range of sizes. Magnetic flattening was achieved in a simple experimental setup in order to deform spheroids and monitor it in real time. By combining these unprecedented magnetic techniques, we observed two different behaviors depending on the size of the spheroids. This Letter provides a tool of choice for remote manipulation of tissues and subsequent investigation of their mechanical properties. Quantitatively, the magnetic force tensiometer provided a simple means of measuring the surface tension of multicellular spheroids, and their elastic modulus. The use of an electromagnet instead of a permanent one will allow us to investigate the frequency-dependent rheology of multicellular spheroids. The method can also be translated to study nonbiological elastoviscoplastic materials. Similarly, active cellular processes (mechanosensing, division, apoptosis, etc), could be explored, and compared to active nonbiological matter.

This work was supported by the French Research Funding Agency (ANR) within the framework of the MagStem (project ID ANR-11-JSV5-0006). We are grateful to Delphine Talbot for providing us with the magnetic nanoparticles, to Nathalie Luciani for her help with the mesenchymal stem cells, and to Cyprien Gay for fruitful discussions.

*Corresponding author.

claire.wilhelm@univ-paris-diderot.fr

[1] O. Campàs, T. Mammoto, S. Hasso, R. a. Sperling, D. O'Connell, A. G. Bischof, R. Maas, D. a. Weitz, L. Mahadevan, and D. E. Ingber, Nat. Methods 11, 183 (2014).

[2] C. J. Miller and L. a. Davidson, Nat. Rev. Genet. 14, 733 (2013). 
[3] L. Liu, G. Duclos, B. Sun, J. Lee, A. Wu, Y. Kam, E. D. Sontag, H. a. Stone, J. C. Sturm, R. a. Gatenby, and R. H. Austin, Proc. Natl. Acad. Sci. U.S.A. 110, 1686 (2013).

[4] J. Folkman and M. Hochberg, J. Exp. Med. 138, 745 (1973).

[5] A. Moscona, Proc. Natl. Acad. Sci. U.S.A. 43, 184 (1957).

[6] R. M. Sutherland, J. a. McCredie, and W. R. Inch, J. Natl. Cancer Inst. (1940-1978) 46, 113 (1971).

[7] M. Vinci, S. Gowan, F. Boxall, L. Patterson, M. Zimmermann, W. Court, C. Lomas, M. Mendiola, D. Hardisson, and S. a. Eccles, BMC Biol. 10, 29 (2012).

[8] D. A. Beysens, G. Forgacs, and J. A. Glazier, Proc. Natl. Acad. Sci. U.S.A. 97, 9467 (2000).

[9] D. Gonzalez-Rodriguez, K. Guevorkian, S. Douezan, and F. Brochard-Wyart, Science 338, 910 (2012).

[10] M. S. Steinberg, Science 141, 401 (1963).

[11] R. A. Foty, G. Forgacs, C. M. Pfleger, and M. S. Steinberg, Phys. Rev. Lett. 72, 2298 (1994).

[12] R. a. Foty and M. S. Steinberg, Dev. Biol. 278, 255 (2005).

[13] M. L. Manning, R. a. Foty, M. S. Steinberg, and E.-M. Schoetz, Proc. Natl. Acad. Sci. U.S.A. 107, 12517 (2010).

[14] T. V. Stirbat, A. Mgharbel, S. Bodennec, K. Ferri, H. C. Mertani, J.-P. Rieu, and H. Delanoë-Ayari, PLoS One 8, e52554 (2013).

[15] J.-L. Maître, H. Berthoumieux, S. F. G. Krens, G. Salbreux, F. Jülicher, E. Paluch, and C.-P. Heisenberg, Science 338, 253 (2012).

[16] H. M. Phillips and M. S. Steinberg, J. Cell Sci. 30, 1 (1978).

[17] G. Forgacs, R. a. Foty, Y. Shafrir, and M. S. Steinberg, Biophys. J. 74, 2227 (1998).

[18] P. Marmottant, A. Mgharbel, J. Käfer, B. Audren, J.-P. Rieu, J.-C. Vial, B. van der Sanden, A. F. M. Marée, F. Graner, and H. Delanoë-Ayari, Proc. Natl. Acad. Sci. U.S.A. 106, 17271 (2009).

[19] T. Vasilica Stirbat, S. Tlili, T. Houver, J.-P. Rieu, C. Barentin, and H. Delanoë-Ayari, Eur. Phys. J. E 36, 84 (2013).

[20] D. Ambrosi and L. Preziosi, Biomech. Model. Mechanobiol. 8, 397 (2009).

[21] H. Byrne and D. Drasdo, J. Math. Biol. 58, 657 (2009).
[22] M. Basan, J. Prost, J.-F. Joanny, and J. Elgeti, Phys. Biol. 8, 026014 (2011).

[23] C. Norotte, F. Marga, a. Neagu, I. Kosztin, and G. Forgacs, Europhys. Lett. 81, 46003 (2008).

[24] A. Mgharbel, H. Delanoë-Ayari, and J.-P. Rieu, HFSP J. 3, 213 (2009).

[25] K. Guevorkian, M.-J. Colbert, M. Durth, S. Dufour, and F. Brochard-Wyart, Phys. Rev. Lett. 104, 218101 (2010).

[26] A. Kalantarian, H. Ninomiya, S. M. I. Saad, R. David, R. Winklbauer, and a. W. Neumann, Biophys. J. 96, 1606 (2009).

[27] R.-Z. Lin, R.-Z. Lin, and H.-Y. Chang, Biotech. J. 3, 1172 (2008).

[28] D. Fayol, G. Frasca, C. Le Visage, F. Gazeau, N. Luciani, and C. Wilhelm, Adv. Mater. 25, 2611 (2013).

[29] T. J. Bartosh, J. H. Ylöstalo, A. Mohammadipoor, N. Bazhanov, K. Coble, K. Claypool, R. H. Lee, H. Choi, and D. J. Prockop, Proc. Natl. Acad. Sci. U.S.A. 107, 13724 (2010).

[30] S. Alimperti, P. Lei, Y. Wen, J. Tian, A. M. Campbell, and S. T. Andreadis, Biotechnology Progress, 30, 974 (2014).

[31] C. Wilhelm and F. Gazeau, Biomaterials 29, 3161 (2008).

[32] D. Fayol, N. Luciani, L. Lartigue, F. Gazeau, and C. Wilhelm, Adv. Healthcare Mater. 2, 313 (2013).

[33] See Supplemental Material at http://link.aps.org/ supplemental/10.1103/PhysRevLett.114.098105 for a summary of the experimental conditions used during the magnetic molding process.

[34] K. Aubertin, S. Bonneau, A. K. a. Silva, J.-C. Bacri, F. Gallet, and C. Wilhelm, PLoS One 8, e84850 (2013).

[35] G. Frasca, V. Du, J.-C. Bacri, F. Gazeau, C. Gay, and C. Wilhelm, Soft Matter 10, 5045 (2014).

[36] P. Aussillous and D. Quéré, Proc. R. Soc. A 462, 973 (2006).

[37] S. C. W. Tan, W. X. Pan, G. Ma, N. Cai, K. W. Leong, and K. Liao, BMC Cell Biol. 9, 40 (2008). 